\title{
Effects of Scattering of Luminescent Down-Shifting Particles Inside Ethylene-Vinyl Acetate Films on Reflection of Light
}

\author{
Shehroz Razzaq *, Ali Asghar and Chaogang Lou* \\ Joint International Research Laboratory of Information Display and Visualization, School of Electronic Science and Engineering,
} Southeast University, Nanjing, China

Silicon solar cells are suffering from a poor spectral response in short wavelength ranges due to the loss of higher energy photons. The luminescent down-shifting (LDS) materials have the ability to convert shorter wavelength photons into longer wavelength photons and improve the performance of the solar cells. However, besides the down-shifting effects, the introduction of LDS particles into ethylene-vinyl acetate (EVA) films on the solar cells also

\section{OPEN ACCESS}

Edited by: Alexey A. Sokol,

University College London, United Kingdom

Reviewed by: Piotr Warszynski, Jerzy Haber Institute of Catalysis and Surface Chemistry, Poland

Rajkumar Kaliyamoorthy, SSN College of Engineering, India

*Correspondence: Shehroz Razzaq 233179974@seu.edu.cn Chaogang Lou lcg@seu.edu.cn

Specialty section:

This article was submitted to Colloidal Materials and Interfaces, a section of the journal Frontiers in Materials

Received: 19 April 2021

Accepted: 26 July 2021

Published: 31 August 2021

Citation:

Razzaq S, Asghar A and Lou C (2021) Effects of Scattering of Luminescent

Down-Shifting Particles Inside

Ethylene-Vinyl Acetate Films on

Reflection of Light.

Front. Mater. 8:697185.

doi: 10.3389/fmats.2021.697185 increases surface reflection, which will negatively influence the performance of the solar cells. In this study, the influence of the size of LDS particles inside EVA films on reflection is investigated theoretically. The results showed that the reflection results from the scattering by LDS particles, which depends on the particle's size. The total reflection caused by LDS particles can be calculated according to the size distribution of LDS particles. This study is helpful in selecting LDS particles that can be applied to solar cells.

Keywords: reflection, solar cells, luminescent down-shifting, eva, particle size distribution

\section{INTRODUCTION}

Crystalline silicon (c-Si) solar cells are the most commonly used photovoltaic devices that convert solar energy into electricity. The maximum theoretical conversion efficiency of c-Si solar cells is around 30\%, which is defined by Shockley and Queisser (1961). One of the factors limiting the efficiency is the spectral mismatch between the incident solar light and the bandgap of Si materials (Huang et al., 2013; Lian et al., 2013; Mambrini et al., 2015; Liu et al., 2017), which results in the poor spectral response of $\mathrm{Si}$ solar cells in short wavelengths $(300-500 \mathrm{~nm})$ due to the energy loss of shortwavelength photons (Shao et al., 2015).

One of the methods to reduce the loss is to use luminescent down-shifting (LDS) materials as a spectral converter transforming one short-wavelength photon into one long-wavelength photon (Richards, 2006). Despite the fact that the quantum yield of the materials does not exceed $100 \%$, this can still be a useful technique to improve the spectral response of $\mathrm{Si}$ solar cells by converting shortwavelength photons into long-wavelength photons (Huang et al., 2013).

The appropriate way to apply the LDS materials to the solar cells is to introduce the LDS particles into ethylene-vinyl acetate (EVA) films, which are located on the top surface of the solar cells. However, the introduced particles in EVA films cause scattering of light and increase surface reflection, which leads to energy loss and offsets the down-shifting effects. So it is important to clarify how these LDS particles influence the light reflection so that we can find a solution to lower the reflection. 




In this study, the simulation on the scatter of LDS particles in the wavelength range from 500 to $1,100 \mathrm{~nm}$ is carried out through the software MiePlot. The effect of LDS particle size on the backscattered power is investigated. The total reflection caused by the scattering is discussed.

\section{SIMULATION}

The simulation starts by calculating the backscattered power of LDS particles with diameters of $5,10,16,22,30$, and $38 \mu \mathrm{m}$. We did not consider the particles larger than $38 \mu \mathrm{m}$ because, if the particle's size continues to increase, such as larger than $50 \mu \mathrm{m}$, the particle's luminescence efficiency will decrease and weaken the downshifting effect. The reflection caused by the scatter is modeled on the basis of the structure shown in Figure 1, which includes EVA films of thickness $300 \mu \mathrm{m}$ and embedded luminescent down-shifting particles with different diameters. Three different particle size distributions (PSD 1, PSD 2, and PSD 3) are used for calculation in this study, as shown in Figure 2 (Huang et al., 2009).

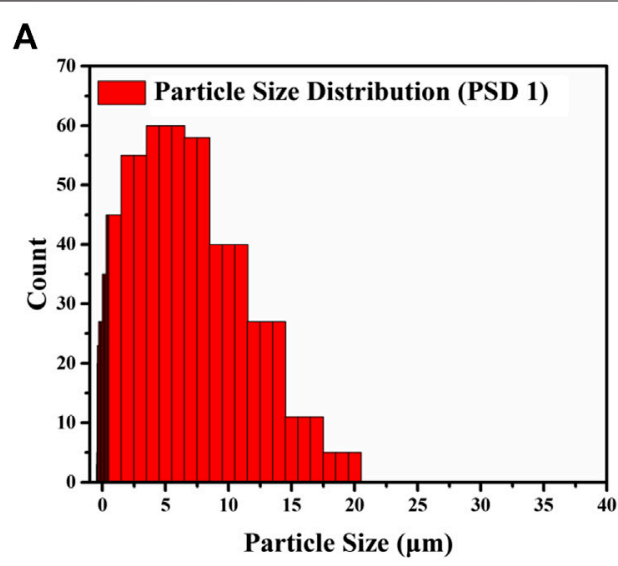

B

\section{C}

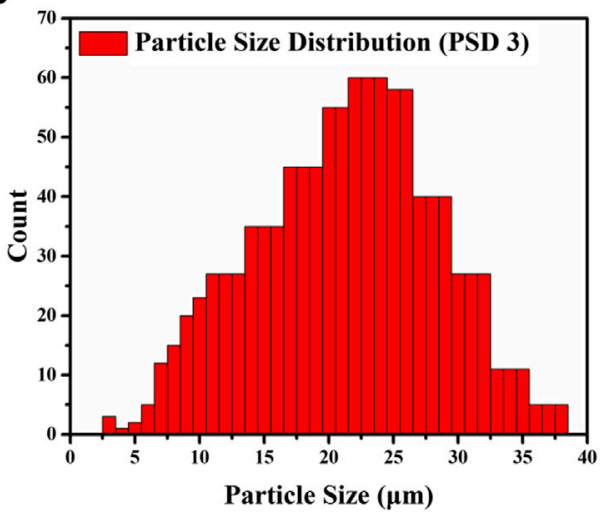

FIGURE 2 | Different particle size distribution of LDS particles in EVA films (A-C). 


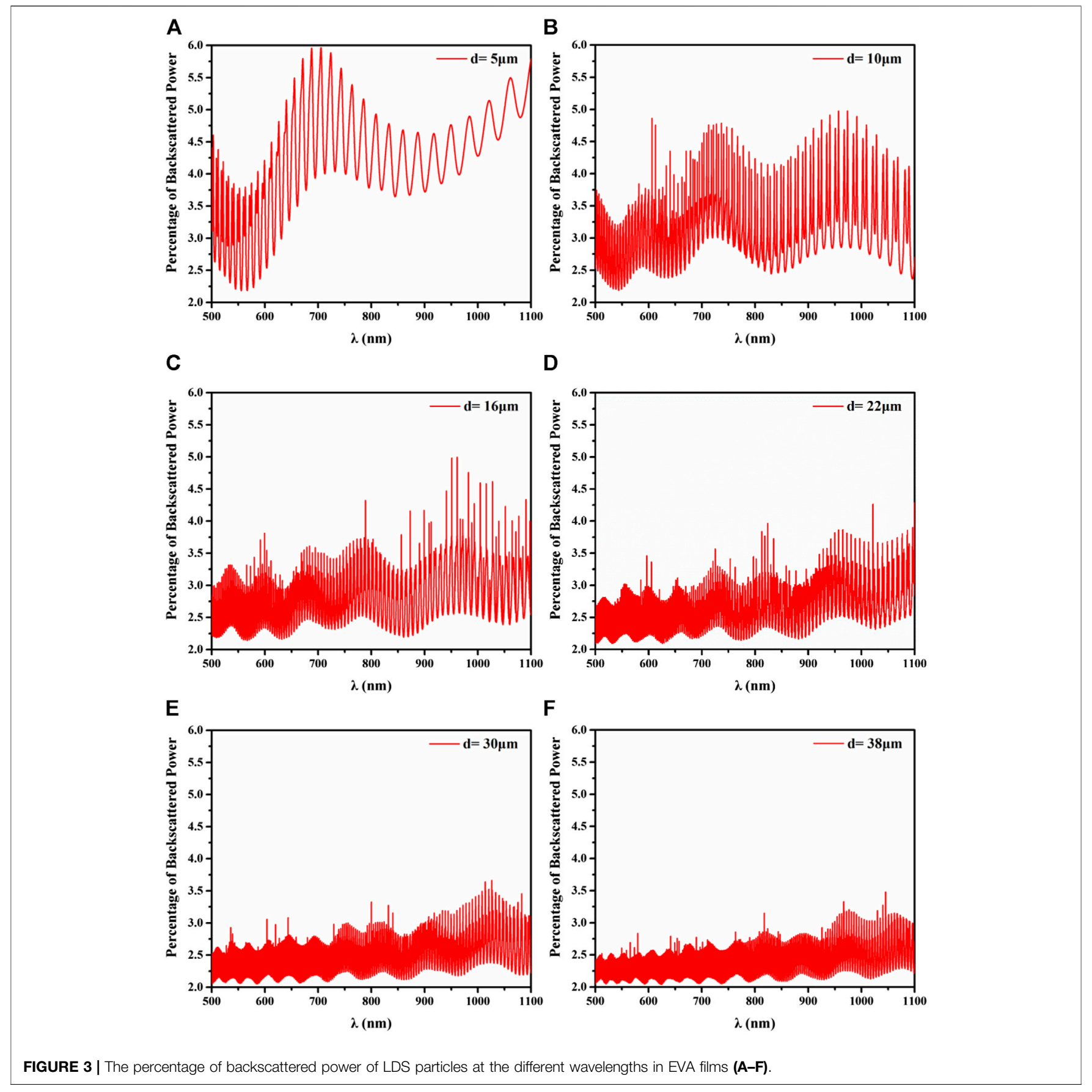

The following conditions are assumed in the simulation: 1) LDS particles are randomly distributed in EVA films; 2) the particles are spherical; and 3) the particles are nonabsorptive. A plane light source with an incident power of $1 \mathrm{~W}$ is placed over the EVA films. The light wavelength is set from 500 to $1,100 \mathrm{~nm}$ because the light with a wavelength shorter than $500 \mathrm{~nm}$ can be absorbed by the particle and has a much more complicated situation. The detector collects the light scattered by the particles in EVA films.

\section{RESULTS AND DISCUSSION}

The percentage of the backscattered power is plotted against incident wavelengths, as shown in Figure 3. It can be seen that the backscattered curves oscillate with the incident wavelength. The percentage of the backscattered power decreases with the particle size and increases with the incident wavelength.

The relationship between the percentage of the backscattered power and the particle size can be explained by the interference 


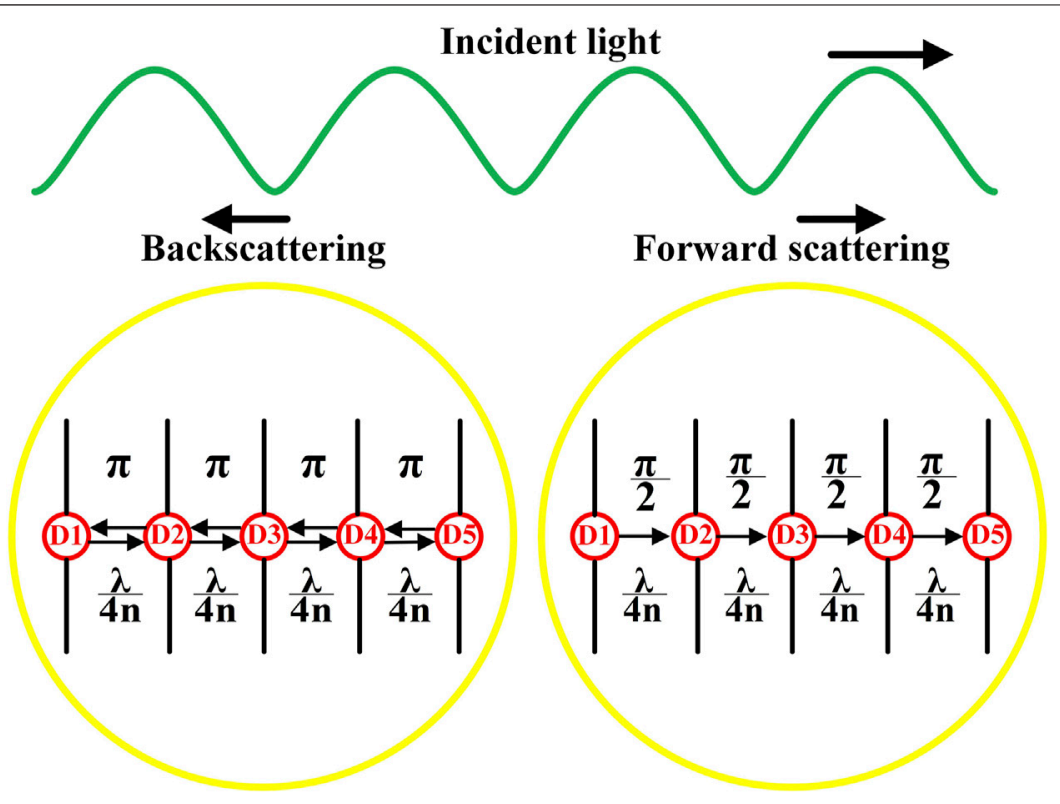

FIGURE 4 | Schematic of light emission by dipoles. D1, D2, D3, D4, and D5 are dipoles.

between the emission from the dipoles inside the particles, as shown in Figure 4 (Razzaq et al., 2021). These results are helpful in choosing the LDS particles with suitable sizes for application in solar cells.

When the particles are excited by incident light, the dipoles inside the particles emit light. For two dipoles with a distance of one-quarter wavelength, the phase difference of the forwardemitted light from these two dipoles is $\frac{\pi}{2}$. While the phase difference of the backward-emitted light from these two dipoles is $\pi$, so their interference is destructive. This indicates that there is more destructive interference in the backward direction than in the forward direction. When the particle size increases, there are more dipoles inside the particle, which leads to more destructive interference in the backward direction. This is why the percentage of the backscattered power decreases with the particle size.

As for the increase in the percentage of the backscattered power with the incident wavelength, it can also be explained by the dipole model shown in Figure 4 (Mie, 1908). When the incident wavelength increases, the refractive indices of the EVA films and LDS particles decrease, respectively. The refractive index is directly related to the dielectric constant of these two materials. When the refractive index of LDS particles decreases, the dielectric constant decreases, leading to a decrease in the density of dipoles. The lower density of dipoles results in an increase in the distance between two dipoles. This means that the number of dipoles whose backward emission interferes destructively decreases, so the percentage of the backscattered power increases with the incident wavelength. This makes the backscattering stronger that raises the total reflection of films.

It can also be seen that the backscattered power of the particles with $30 \mu \mathrm{m}$ is similar to that with $38 \mu \mathrm{m}$. This can be attributed to

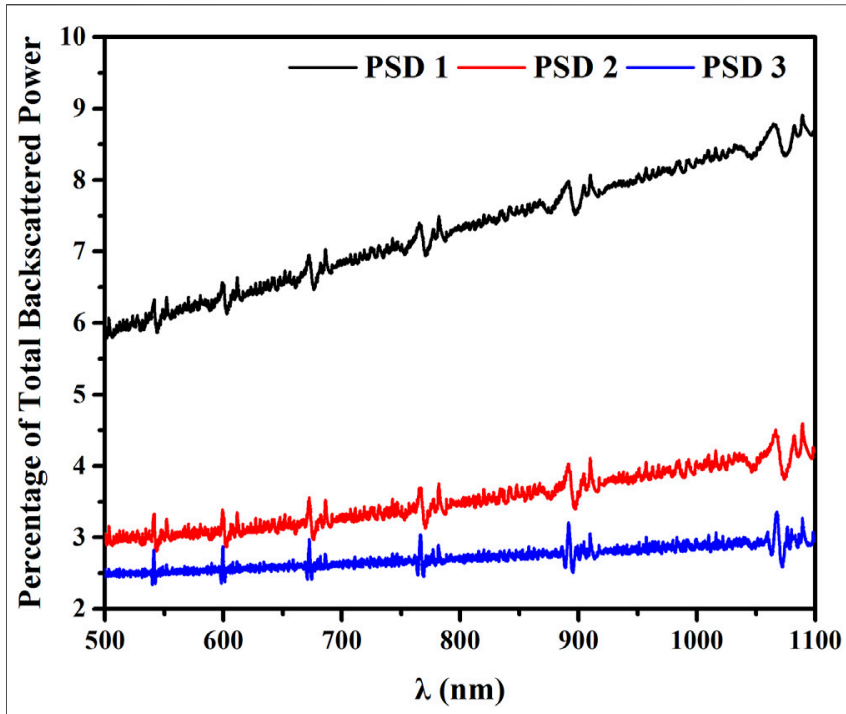

FIGURE 5|Percentage of total backscattered power of different particle size distributions.

the light's penetration depth. For the particles with sizes 30 and $38 \mu \mathrm{m}$, most of the emission from the particles comes from the dipoles near the light source, and the emission from the dipoles at the other end of the particles is much weaker. When the particle is small, with the increasing size, more dipoles are excited, and the scattering becomes stronger. When the particle's size reaches around the penetration depth (in our simulation, the penetration depth is about $22 \mu \mathrm{m}$ ), the scattering intensity becomes the strongest. If the particle's size continues to rise, 


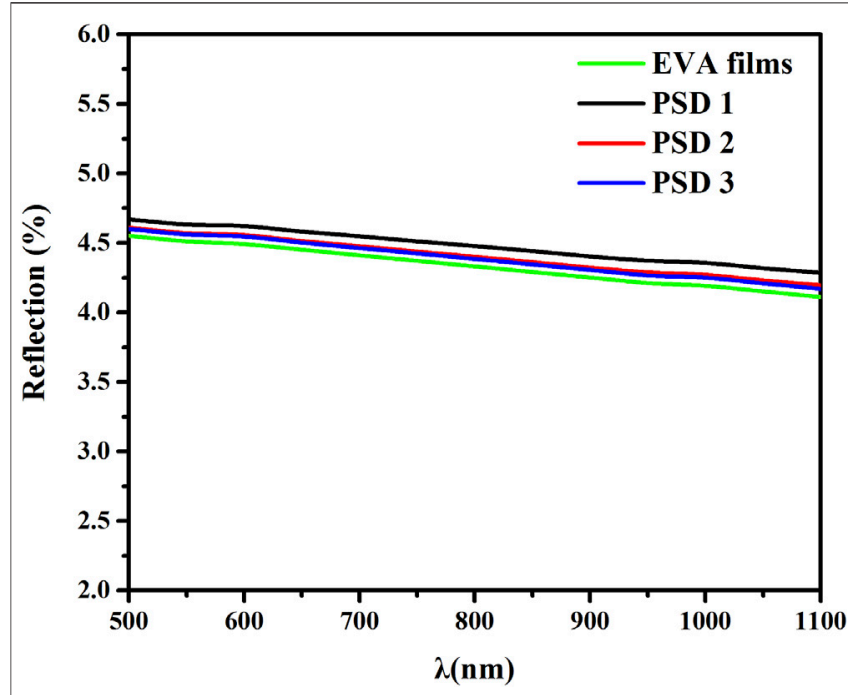

FIGURE 6 | Reflection of EVA films with different particle size distributions of LDS particles.

the increase of the scattering intensity becomes slow. So the increase of the backscattered power also becomes slow. This is why the difference between the backscattered powers of the particles with $35 \mu \mathrm{m}$ and that with $38 \mu \mathrm{m}$ is small, as shown in Figure 3.

Figure 5 shows the total backscattered power of different size distributions of LDS particles in EVA films. It can be seen that the backscattered power increases with the incident wavelength. This means that, although the LDS particles can improve the response of the solar cells in the short wavelength range, the reflection caused by their scattering in the middle- and long-wavelength range may deteriorate the cell's performance.

Figure 6 shows the total reflection of EVA films with and without LDS particles. The reflection of EVA films is calculated by using the Fresnel equation. To calculate the reflection of films with PSDs of the LDS particle, it is assumed that all the particles are on the same level. Because the density of the particles in EVA films is very low (the mass ratio of EVA to phosphors is 100:0.3), the overlapping of LDS particles has been ignored. Clearly, the introduction of LDS particles increases with the wavelength that leads to an increase in the reflection.

The reflection caused by LDS particles plays an important role in the performance of solar cells. The reason is that the improvement caused by LDS in UV/blue range is slight, and if it cannot compensate for the increased reflection in the visible

\section{REFERENCES}

Huang, C. K., Chen, Y. C., Hung, W. B., Chen, T. M., Sun, K. W., and Chang, W. L. (2013). Enhanced Light Harvesting of Si Solar Cells via Luminescent Down Shifting Using YVO4: Bi3+, Eu3+ Nanophosphors. Prog. Photovoltaics 21, 1507-1513. doi:10.1002/pip.2222

Huang, S. C., Wu, J. K., Hsu, W. J., Chang, H. H., Hung, H. Y., Lin, C. L., et al. (2009). Particle Size Effect on the Packaging Performance of YAG:Ce Phosphors in white LEDs. Int. J. Appl. Ceram. Technol. 6, 465-469. doi:10.1111/j.1744-7402.2009.02367.x and near-infrared range, the conversion efficiency of the solar cells will decrease. Therefore, finding the suitable size of phosphor particles and suppressing the scattering-induced reflection is important.

Besides, the dependence of the backscattering on the particle size, the down-shifting effect also depends on the particle size. If the particle size continues to increase, such as larger than $38 \mu \mathrm{m}$, the particle's luminescence efficiency will decrease and weaken the down-shifting effect. This is because when the particle size is bigger than the penetration depth, the emission from the particles hardly increases. There thus exists a suitable size of LDS particles that results in better improvement in the performance of the solar cells.

\section{CONCLUSION}

The influence of the luminescent down-shifting particles inside EVA films on reflection has been studied numerically. Simulation results show that the reflection increases due to the scattering of LDS particles. The percentage of the backscattered power decreases with the particle size and increases with the incident wavelength, which can be explained by the dipole models. The reflection caused by the particles offsets their down-shifting effect. To improve the performance of solar cells, it is necessary to find the LDS particle with a suitable size.

\section{DATA AVAILABILITY STATEMENT}

The original contributions presented in the study are included in the article/Supplementary Material; further inquiries can be directed to the corresponding author.

\section{AUTHOR CONTRIBUTIONS}

The authors confirm being the sole contributors of this work and have approved it for publication.

\section{FUNDING}

The authors thank the supports from the Natural Science Foundation of Jiangsu (Grant No. BK2011033) and the Primary Research and Development Plan of Jiangsu Province (Grant No. BE2016175).

Huang, X., Han, S., Huang, W., and Liu, X. (2013). Enhancing Solar Cell Efficiency: The Search for Luminescent Materials as Spectral Converters. Chem. Soc. Rev. 42, 173-201. doi:10.1039/c2cs35288e

Lian, H., Hou, Z., Shang, M., Geng, D., Zhang, Y., and Lin, J. (2013). Rare Earth Ions Doped Phosphors for Improving Efficiencies of Solar Cells. Energy 57, 270-283. doi:10.1016/j.energy.2013.05.019

Liu, W., Zhang, J., Hao, Z., Xiang, G., Zhang, L. L., Zhang, X., et al. (2017). Near-infrared Quantum Cutting and Energy Transfer Mechanism in Lu2O3: Tm3+/Yb3+ Phosphor for High-Efficiency Photovoltaics. 
J. Mater. Sci. Mater. Electron. 28, 8017-8022. doi:10.1007/s10854-0176506-5

Mambrini, T., Dubois, A. M., Longeaud, C., Badosa, J., Haeffelin, M., Prieur, L., et al. (2015). Photovoltaic Yield: Correction Method for the Mismatch between the Solar Spectrum and the Reference ASTMG AM1.5G Spectrum. EPJ Photovoltaics 6. doi:10.1051/epjpv/2014011

Mie, G. (1908). Beiträge zur Optik trüber Medien, speziell kolloidaler Metallösungen. Ann. Phys. 330, 377-445. doi:10.1002/andp.19083300302

Razzaq, S., Lou, C., and Asghar, A. (2021). Forward and Back Scattering of Luminescent Down-Shifting Particles in EVA Films. 10-13. doi:10.1109/GreenTech48523.2021.00015

Richards, B. S. (2006). Luminescent Layers for Enhanced Silicon Solar Cell Performance: Down-Conversion. Solar Energ. Mater. Solar Cell 90, 1189-1207. doi:10.1016/j.solmat.2005.07.001

Shao, G., Lou, C., and Xiao, D. (2015). Enhancing the Efficiency of Solar Cells by Down Shifting YAG: Ce3+ Phosphors. J. Lumin. 157, 344-348. doi:10.1016/j.jlumin.2014.08.064 Shockley, W., and Queisser, H. J. (1961). Detailed Balance Limit of Efficiency of P-N junction Solar Cells. J. Appl. Phys. 32, 510-519. doi:10.1063/1.1736034
Conflict of Interest: The authors declare that the research was conducted in the absence of any commercial or financial relationships that could be construed as a potential conflict of interest.

Publisher's Note: All claims expressed in this article are solely those of the authors and do not necessarily represent those of their affiliated organizations, or those of the publisher, the editors, and the reviewers. Any product that may be evaluated in this article, or claim that may be made by its manufacturer, is not guaranteed or endorsed by the publisher.

Copyright (c) 2021 Razzaq, Asghar and Lou. This is an open-access article distributed under the terms of the Creative Commons Attribution License (CC $B Y)$. The use, distribution or reproduction in other forums is permitted, provided the original author(s) and the copyright owner(s) are credited and that the original publication in this journal is cited, in accordance with accepted academic practice. No use, distribution or reproduction is permitted which does not comply with these terms. 